\title{
Cyclostationarity-based Doppler Spread Estimation in Mobile Fading Channels
}

Hong Zhang, Student Member, IEEE, and Ali Abdi, Senior Member, IEEE

\begin{abstract}
The Doppler spread, or equivalently, the mobile speed, is a measure of the spectral dispersion of mobile fading channel. Accurate estimation of the mobile speed is of importance in wireless mobile applications which require the knowledge of the rate of channel variations. This paper exploits the inherent cyclostationarity of linearly modulated signals, transmitted through fading channels, to design robust blind and data-aided mobile speed estimators. Two categories of cyclic-correlation- and cyclicspectrum-based methods are developed. Extension to space-time speed estimation at the base station in macrocells is also provided. In comparison with the existing methods, the new estimators can be used without any need for pilot tones, and are robust to additive stationary noise or interference of any color or distribution. Unlike the conventional multi-antenna based method, the proposed space-time speed estimator does not assume the receiver noise to be spatially white. We also devised a suboptimal training sequence for pilot-symbol assisted methods, to reduce the estimation error. The performance of the proposed estimators are illustrated via extensive Monte Carlo simulations.
\end{abstract}

\section{Index Terms}

Cyclostationary, Doppler estimation, velocity estimation, fading channels, multipath channels, cyclic correlation, cyclic spectrum, Doppler spectrum, multiple antennas, macrocell.

\section{INTRODUCTION}

In mobile communication systems, the received signal strength varies significantly in time due to the constructive and destructive superpositions of the replicas of the transmitted signal, in

This work was presented in part at IEEE Global Telecommunications Conference, San Francisco, CA, 2006.

H. Zhang and A. Abdi are with Center for Wireless Communications and Signal Processing Research (CWCSPR), Department of Electrical and Computer Engineering, New Jersey Institute of Technology, Newark, NJ 07012 USA (emails: hz7@njit.edu, ali.abdi@njit.edu) 
multipath channels. In addition, the motion of mobile station (MS) results in the Doppler shifts of different frequencies. The Doppler spread, which corresponds to the maximum Doppler shift and is proportional to the mobile speed, dictates how fast the fading changes. Knowledge of the Doppler spread is useful in a number of mobile communication subsystems.

One important application is adaptive transceiver design. In order to improve performance or reduce complexity, the system parameters need to be adapted to the changing channel conditions [1], which in turn necessitates the estimation of Doppler spread. For example, the MS can use Doppler information to regularly adjust the power of the received signal to exploit, as much as possible, the dynamic range of $\mathrm{A} / \mathrm{D}$ converters. This is an important power control issue in CDMA-based mobile communication systems. Channel estimation algorithms can also benefit from the knowledge of the mobile speed. Examples include the optimization of the channel tracker step size and adaption of the complexity of the algorithm in adaptive equalizers. Other applications such as variable-rate coding and interleaving can take advantage of the Doppler information as well.

Knowledge of the mobile speed is also useful for cellular network control algorithms, such as handoff and channel/cell assignment [2]. Handoff algorithms normally use the average strength of the received signal to determine when the MS is to be served by another base station (BS). Appropriate choice of a the temporal averaging window length for signal strength estimation is dependent on the mobile speed. To significantly reduce the number of unnecessary handoffs in an area with both slow- and fast-moving mobiles, the channel/cell assignment algorithm uses the estimated mobiles' speeds to allocate slow mobiles to microcells and fast ones to macrocells.

There are four major classes of speed estimation techniques: crossing-based methods [2] [3], covariance-based methods [4] [5] [6] [7] [8], maximum likelihood (ML) based methods [9] [10], and power spectrum density (PSD) based methods [11] [12] [13]. Crossing-based approaches rely on the number of received signal level crossing, which is proportional to the mobile speed. On the other hand, covariance-based algorithms exploit the Doppler information which exists in the sample autocovariance of the received signal. Finally, the newly developed PSD based estimators utilize some unique features of the Doppler spectrum.

All the above existing speed estimators work based on transmitted pilot tones. To the best of our knowledge, only one [14] has addressed blind speed estimation in a nonlinear modulation system, with no analytical or simulation-based performance evaluation. In this paper, we develop 
new speed estimators by exploiting the cyclostationarity of linearly modulated signals. The new estimators can be applied blindly, without using training symbols, which increase the data throughput. On the other hand, for the case where pilot symbols are available, we have proposed novel estimators which provide more accurate estimates, compared to blind methods. Essentially, cyclostationarity-based estimators are not sensitive to additive stationary noise or interference of any color or distribution, which are known to affect pilot-tone-based approaches. The robustness of the new estimators to nonisotropic scattering and the variations of line-of-sight (LOS) is also investigated. Extensive Monte Carlo simulations are conducted to illustrate the performance of the new estimators.

The organization of this paper is as follows. The signal, channel, and noise models are discussed in Section II, whereas the cyclostationarity of linearly modulated waveforms is briefly reviewed in Section III. The new single-antenna speed estimators are presented in Section IV, extension to systems with multiple antennas is addressed in Section V. Section VI provides the numerical results and Section VII concludes the paper.

\section{Signal, Channel, And Noise Models}

The received lowpass complex envelope of a linearly modulated signal, transmitted through a frequency-flat fading channel, can be expressed as [15]

$$
z(t)=h(t) s(t)+v(t)
$$

where $s(t)=\sum_{m} w(m) g(t-m T)$, and we have the following definitions

$h(t)$ fading channel,

$w(m)$ stationary random sequence of transmitted symbols chosen from a finite-alphabet complex constellation,

$g(t)$ convolution of the transmitter's signaling pulse and the receiver filter which, without loss of generality, is a raised cosine with rolloff factor $\beta \in[0,1][15]$,

$v(t)$ a complex stationary process which represents the summation of noise and interference, independent of $h(t)$ and $w(m)$,

T symbol period. 
The unit-power fading process $h(t)$ includes the random diffuse component $h^{d}(t)$ and the deterministic LOS component

$$
h(t)=\sqrt{\frac{1}{K+1}} h^{d}(t)+\sqrt{\frac{K}{K+1}} e^{-j 2 \pi f_{D} t \cos \alpha_{0}+j \varphi_{0}} .
$$

In eq. (2), $h^{d}(t)$ is a zero-mean unit-variance stationary complex Gaussian process and the Rician factor $K$ is the ratio of the LOS power to the diffuse power. In the LOS component we have $f_{D}=\nu / \lambda=\nu f_{c} / c$ as the maximum Doppler frequency in $\mathrm{Hz}, \nu$ is the mobile speed, $\lambda$ is the wavelength, $f_{c}$ is the carrier frequency, and $c$ is the speed of light. Furthermore, $j^{2}=-1$, and $\alpha_{0}$ and $\varphi_{0}$ stand for the angle-of-arrival (AOA) and the phase of the LOS component at the receiver, respectively.

With von Mises distribution for the AOA, the autocorrelation function of $h(t)$, defined by $r_{h}(\tau)=E\left[h(t) h^{*}(t+\tau)\right]$, where $E[$.$] and *$ denote mathematical expectation and complex conjugate, respectively, is given by [16]

$$
\begin{aligned}
r_{h}(\tau)= & \frac{K}{K+1} \exp \left(j 2 \pi f_{D} \tau \cos \alpha_{0}\right)+\frac{1}{K+1} \\
& \times \frac{I_{0}\left(\sqrt{\kappa^{2}-4 \pi f_{D}^{2} \tau^{2}+j 4 \pi \kappa f_{D} \tau \cos \alpha}\right)}{I_{0}(\kappa)},
\end{aligned}
$$

where $\alpha \in[-\pi, \pi)$ is the mean AOA of the diffuse component, $\kappa \geq 0$ controls the width of the diffuse component AOA, and $I_{0}($.$) is the zero-order modified Bessel function of the first kind. Eq.$ (3) is an empirically-verified extension of the well-known Clarkes' model $r_{h}(\tau)=J_{0}\left(2 \pi f_{D} \tau\right)$ $[2]$.

\section{Cyclostationarity of the ReCEIVEd SignAl}

The time-varying autocorrelation of the received signal $z(t)$, defined by $r_{z}(t ; \tau)=r_{z}(t, u)=$ $E\left[z(t) z^{*}(u)\right]$ with $u=t+\tau$, can be shown to be

$$
r_{z}(t ; \tau)=r_{h}(\tau) r_{s}(t ; \tau)+r_{v}(\tau)
$$

where

$$
\begin{aligned}
r_{s}(t ; \tau) & =\sum_{m=-\infty}^{\infty} \sum_{n=-\infty}^{\infty} \tilde{r}_{w}(n-m) g(t-m T) g^{*}(t+\tau-n T) \\
& =\sum_{l=-\infty}^{\infty} \tilde{r}_{w}(l) \sum_{m=-\infty}^{\infty} g(t-m T) g^{*}(t+\tau-l T-m T)
\end{aligned}
$$


in which $\tilde{r}_{w}(n-m)=E\left\{w(m) w^{*}(n)\right\}$ and $l=n-m$. It is well known that $s(t)$ is a cyclostationary random process since $r_{s}(t ; \tau)$ is periodic in $t$, with period $T$ [17]. Consequently we have $r_{z}(t+k T ; \tau)=r_{z}(t ; \tau), \forall t, \tau$, which indicates that $z(t)$ is cyclostationary as well, with the same period $T$.

The cyclic correlations which are the Fourier coefficients of $r_{z}(t ; \tau)$ for cyclic frequencies $k / T=0, \pm 1 / T, \pm 2 / T, \ldots$, are given by [17]

$$
\begin{aligned}
R_{z}(k ; \tau) & =\frac{1}{T} \int_{0}^{T} r_{z}(t ; \tau) \exp \{-j 2 \pi k t / T\} d t \\
& =r_{h}(\tau) R_{s}(k ; \tau)+r_{v}(\tau) \delta_{k}
\end{aligned}
$$

where $\delta_{k}$ is the Kronecker delta function, which is 1 when $k=0$ and 0 otherwise. Furthermore, $R_{s}(k ; \tau)$ is cyclic correlation of $s(t)$, which is the inverse Fourier transform of $s(t)$ 's cyclic spectrum $\Omega_{s}(k ; f)$, given by [17]

$$
\Omega_{s}(k ; f)=\frac{1}{T} G(f) G^{*}\left(f-\frac{k}{T}\right) \tilde{\Omega}_{w}(f),
$$

where $G(f)$ is the Fourier transform of $g(t)$ and $\tilde{\Omega}_{w}(f)$ is the power spectrum of the sequence of transmitted symbols $\{w(m)\}$, which is also the Fourier transform of $\tilde{r}_{w}(l)$ and given by

$$
\tilde{\Omega}_{w}(f)=\sum_{l=-\infty}^{\infty} \tilde{r}_{w}(l) e^{-j 2 \pi l T f} .
$$

\section{The CyCLOSTATIONARITY-BASED ESTIMATORS}

\section{A. The Cyclic Correlation Approach}

For an arbitrary proper complex process $z(t)$ [18], it is known that $r_{\dot{z}}(t ; \tau)=r_{\dot{z}}(t, u)=$ $\partial^{2} r_{z}(t ; u) / \partial t \partial u$, where dot denotes differentiation with respect to time $t$. According to (4) we have

$$
\begin{aligned}
r_{\dot{z}}(t ; \tau)= & r_{\dot{h}}(\tau) r_{s}(t ; \tau)+r_{h \dot{h}}(\tau) r_{\dot{s} s}(t ; \tau)+r_{\dot{h} h}(\tau) r_{s \dot{s}}(t ; \tau) \\
& +r_{h}(\tau) r_{\dot{s}}(t ; \tau)+r_{\dot{v}}(\tau)
\end{aligned}
$$

where $r_{h \dot{h}}(\tau)=E\left\{h(t) \dot{h}^{*}(t+\tau)\right\}, r_{\dot{h} h}(\tau)=E\left\{\dot{h}(t) h^{*}(t+\tau)\right\}, r_{s \dot{s}}(t ; \tau)=E\left\{s(t) \dot{s}^{*}(t+\tau)\right\}$, $r_{\dot{s} s}(t ; \tau)=E\left\{\dot{s}(t) s^{*}(t+\tau)\right\}$, and

$$
r_{\dot{s}}(t, \tau)=\sum_{m=-\infty}^{\infty} \sum_{n=-\infty}^{\infty} \tilde{r}_{w}(n-m) \dot{g}(t-m T) \dot{g}^{*}(t+\tau-n T) .
$$


It is easy to show that $r_{s \dot{s}}(t ; \tau), r_{\dot{s} s}(t ; \tau)$ and $r_{\dot{s}}(t, \tau)$ are periodic w.r.t. $t$ with period $T$. Therefore $\dot{z}(t)$ is cyclostationary as well, with the same period $T$. With the assumption of isotropic scattering and no LOS, i.e., $r_{h}(\tau)=J_{0}\left(2 \pi f_{D} \tau\right)$ where $J_{0}($.$) is the zero-order Bessel function of$ the first kind, one obtains $r_{h \dot{h}}(0)=r_{\dot{h} h}(0)=0$ and $r_{\dot{h}}(0)=-\left.r_{h}^{\prime \prime}(\tau)\right|_{\tau=0}=2 \pi^{2} f_{D}^{2}$, where prime denotes differentiation with respect to $\tau$. Therefore, the cyclic correlation of $\dot{z}(t)$ at $\tau=0$ can be obtained by calculating the $k$-th Fourier coefficients of (9) w.r.t. $t$

$$
R_{\dot{z}}(k ; 0)=2 \pi^{2} f_{D}^{2} R_{s}(k ; 0)+R_{\dot{s}}(k ; 0)+r_{\dot{v}}(0) \delta_{k},
$$

where $R_{s}(k ; 0)$ and $R_{\dot{s}}(k ; 0)$ are the $k$-th Fourier coefficients of (5) and (10), respectively.

To obtain a noise free estimator for $f_{D}$, we choose $k \neq 0$ and divide $R_{\dot{z}}(k ; 0)$ in (11) by $R_{z}(k ; 0)$ in $(6)$, which after rearranging the terms gives us

$$
f_{D}^{2}=\frac{1}{2 \pi^{2}}\left(\frac{R_{\dot{z}}(k ; 0)}{R_{z}(k ; 0)}-\frac{R_{\dot{s}}(k ; 0)}{R_{s}(k ; 0)}\right), k \neq 0
$$

The cyclic correlations of $z(t)$ can be estimated from the discrete-time-version signal of $z(t)$, oversampled at a rate of $P / T$ and represented by $\{z[n]\}_{n=0}^{N-1}[19]$

$$
\hat{R}_{z}\left(k ; P \tau_{d} / T\right)=\frac{1}{\sqrt{N}} \sum_{n=0}^{N-\tau_{d}-1} z[n] z^{*}\left[n+\tau_{d}\right] e^{-j 2 \pi k n / P},
$$

where $\tau_{d}=0,1,2, \ldots, N-1$. To estimate the cyclic correlations of $\dot{z}(t)$, first $\dot{z}(t)$ can be estimated from the oversampled version of $z(t)$ via a finite difference approximations, which yields $\{\dot{z}[n]\}_{n=0}^{N-1}$. Then, similar to (13) we have

$$
\hat{R}_{\dot{z}}\left(k ; P \tau_{d} / T\right)=\frac{1}{\sqrt{N}} \sum_{n=0}^{N-\tau_{d}-1} \dot{z}[n] \dot{z}^{*}\left[n+\tau_{d}\right] e^{-j 2 \pi k n / P} .
$$

Note that $R_{s}(k ; 0)$ and $R_{\dot{s}}(k ; 0)$ in (12) depend on the statistics of the symbols $\{w(m)\}$, as well as the pulse shape.

1) Blind Speed Estimator: Assuming the transmitted data symbol sequence $\{w(m)\}$ is white and zero-mean, eq. (7) simplifies to [17]

$$
\Omega_{s}(k ; f)=\frac{\sigma_{w}^{2}}{T} G(f) G^{*}\left(f-\frac{k}{T}\right),
$$

where $\sigma_{w}^{2}=E\left[|w(m)|^{2}\right]$ is the average power of the sequence. It is easy to see that $\Omega_{s}(k ; f)=0$, $\forall|k| \geq 2$, when $g(t)$ is a raised cosine. By taking the inverse Fourier transform of (15), one 
can show that

$$
\begin{aligned}
R_{s}(k ; \tau)= & \frac{\sigma_{w}^{2}}{T} e^{j \pi k \tau / T} \int_{-\infty}^{\infty} G\left(f+\frac{k}{2 T}\right) \\
& \times G^{*}\left(f-\frac{k}{2 T}\right) e^{-j 2 \pi f \tau} d f
\end{aligned}
$$

which results in

$$
\begin{aligned}
R_{s}(1 ; \tau) & =\frac{\sigma_{w}^{2} T}{4} e^{j \pi \tau / T} \int_{-\beta /(2 T)}^{\beta /(2 T)} \cos ^{2}\left(\frac{\pi f T}{\beta}\right) e^{-j 2 \pi f \tau} d f \\
& =\frac{\sigma_{w}^{2} T^{3} \sin (\pi \beta \tau / T)}{8 \pi\left(T^{2} \tau-\beta^{2} \tau^{3}\right)} e^{j \pi \tau / T}
\end{aligned}
$$

Based on (10) and (15), we have

$$
\Omega_{\dot{s}}(k ; f)=\sigma_{w}^{2} T^{-1} \breve{G}(f) \breve{G}^{*}(f-k / T),
$$

where

$$
\breve{G}(f)=j 2 \pi f G(f)
$$

Similar to (16), one obtains

$$
\begin{aligned}
R_{\dot{s}}(k ; \tau)= & \frac{\sigma_{w}^{2}}{T} e^{j \pi k \tau / T} \int_{-\infty}^{\infty} \breve{G}\left(f+\frac{k}{2 T}\right) \\
& \times \breve{G}^{*}\left(f-\frac{k}{2 T}\right) e^{-j 2 \pi f \tau} d f
\end{aligned}
$$

which gives us

$$
\begin{aligned}
R_{\dot{s}}(1 ; \tau)= & \int_{-\beta /(2 T)}^{\beta /(2 T)}\left(f^{2}-\frac{1}{4 T^{2}}\right) \cos ^{2}\left(\frac{\pi f T}{\beta}\right) e^{-j 2 \pi f \tau} d f \\
& \times \sigma_{w}^{2} T \pi^{2} e^{j \pi \tau / T}
\end{aligned}
$$

By substituting (17) and (21) into (12), the blind speed estimator can be written as

$$
\hat{f}_{D}=\frac{1}{\sqrt{2} \pi} \sqrt{\frac{\hat{R}_{\dot{z}}(1 ; 0)}{\hat{R}_{z}(1 ; 0)}-\frac{R_{\dot{s}}(1 ; 0)}{R_{s}(1 ; 0)}}
$$

where

$$
\frac{R_{\dot{s}}(1 ; 0)}{R_{s}(1 ; 0)}=\frac{\left(\pi^{2}-6\right) \beta^{2}-3 \pi^{2}}{3 T^{2}},
$$

obtained from (17) and (21). 
2) Data-Aided Speed Estimator: It has already been noted that $R_{s}(k ; \tau)$, and therefore $R_{z}(k ; \tau)$, depend on the transmitted symbol sequence and the rolloff factor of the pulse shape. This indicates that there may exist an optimal training sequence to improve the performance of cyclostationarity-based estimators. In general, the optimal solution is not easy to obtain due to the highly nonlinear form of the estimator in (22). However, it is possible to choose a specific sequence to obtain a convenient estimator. For example, if we choose the fixed training sequence $w(m)=(-1)^{m} \sigma_{w}$ where $\sigma_{w}$ is a constant, in Appendix I it is shown that

$$
\begin{gathered}
R_{s}(1 ; \tau)=\frac{\sigma_{w}^{2}}{4} \exp \{j \pi \tau / T\}, \\
R_{\dot{s}}(1 ; \tau)=-\frac{\pi^{2} \sigma_{w}^{2}}{4 T^{2}} \exp \{j \pi \tau / T\},
\end{gathered}
$$

which interestingly do not depend on the rolloff factor $\beta$. Following the same approach that resulted in (22) and (23), the data-aided speed estimator can be derived as

$$
\hat{f}_{D}=\frac{1}{\sqrt{2} \pi} \sqrt{\frac{\hat{R}_{\dot{z}}(1 ; 0)}{\hat{R}_{z}(1 ; 0)}+\frac{\pi^{2}}{T^{2}}}
$$

Remark 1: With $R_{s}^{-1}(1 ; \tau)$ given in (24), one can recover the fading channel correlation from (6), i.e., $r_{h}(\tau)=R_{s}^{-1}(1 ; \tau) R_{z}(1 ; \tau)$. With this estimate of $r_{h}(\tau)$ one can use correlation matching [3], polynomial solving [7], or any other technique which estimates the Doppler spread directly from $r_{h}(\tau)$.

\section{B. The Cyclic Spectrum Approach}

By taking the Fourier transform of (6) w.r.t. $\tau$, for $k \neq 0$, we obtain

$$
\Omega_{z}(k ; f)=\Omega_{h}(f) \circledast \Omega_{s}(k ; f), \quad k \neq 0,
$$

where $\circledast$ denotes convolution. Note that by choosing $k \neq 0$, the effect of noise is disappeared. Now the idea is to obtain $f_{D}$ from the estimate of $\Omega_{z}(k ; f)$. The consistent cyclic spectrum

estimate can be obtained by windowing $\hat{R}_{z}\left(k ; P \tau_{d} / T\right)$ in (13) with the window $W^{\left(2 L_{g}+1\right)}\left(\tau_{d}\right)$, defined over $\left[-L_{g}, L_{g}\right][19]$

$$
\begin{aligned}
\hat{\Omega}_{z}\left(k ; P f_{d} / T\right)= & \frac{T}{P} \sum_{\tau_{d}=-L_{g}}^{L_{g}} W^{\left(2 L_{g}+1\right)}\left(\tau_{d}\right) \hat{R}_{z}\left(k ; P \tau_{d} / T\right) \\
& \times \exp \left\{-j 2 \pi f_{d} \tau_{d}\right\} .
\end{aligned}
$$


1) Data-Aided Speed Estimator: Based on (24), $\Omega_{s}(1 ; f)$ is an impulse at $f=1 /(2 T)$. Therefore $\Omega_{z}(1 ; f)$ is simply the Doppler spectrum $\Omega_{h}(f)$ shifted from $f=0$ to $f=1 /(2 T)$. In other words, $\Omega_{z}(1 ; f)$ has two strong peaks at $f=1 /(2 T) \pm f_{D}$ due to the singularities presented in $\Omega_{h}(f)$. Hence, we can use the same technique as in [13] to estimate $f_{D}$, i.e.

$$
\hat{f}_{D}=\frac{P}{T}\left|-\frac{1}{2 T}+\arg \max _{f}\right| \hat{\Omega}_{z}(1 ; f)|| .
$$

Remark 2: This peak detection approach for the data-aided estimator can not be extended to blind speed estimation in general. This is because the convolution in (27) in general will not result in a power spectrum with a peak that facilitates the estimation. However, in some special cases such as $\beta=0,(29)$ is applicable to the blind method.

\section{A Multi-Antenna Cyclostationarity-BAsed Estimator}

Multi-antenna systems can take advantage of the cyclostationarity of modulated signals [20]. In this section, we extend our algorithm to a system with multiple antennas, to improve the performance. Consider a uniform linear antenna array at an elevated base station (BS) of a marcocell, composed of $L$ omnidirectional unit-gain elements, with element spacing $d$. The BS experiences no local scattering, whereas the single-antenna mobile station (MS) is surrounded by local scatters. Let the received signals at the $l$-th element be $z_{l}(t)=h_{l}(t) s(t)+v_{l}(t), l=$ $1,2, \ldots, L$, which is similar to (1). Then the time-varying space-time crosscorrelation function between $z_{a}(t)$ and $z_{b}(t), 1 \leq a \leq b \leq L$, defined by $r_{z}((b-a) \Delta,(t ; \tau))=E\left[z_{a}(t) z_{b}^{*}(t+\tau)\right]$ such that $\Delta=d / \lambda$, is given by

$$
r_{z}((b-a) \Delta,(t ; \tau))=r_{h}((b-a) \Delta, \tau) r_{s}(t ; \tau)+r_{v}(\tau) \delta_{b-a}
$$

where the space-time channel crosscorrelation, defined by $r_{h}((b-a) \Delta, \tau)=E\left[h_{a}(t) h_{b}^{*}(t+\tau)\right]$, is given by [21]

$$
\begin{gathered}
r_{h}((b-a) \Delta, \tau)=\frac{K}{K+1} \exp \left(j(\zeta+\varrho) \cos \alpha_{0}\right)+\frac{1}{K+1} \\
\times \frac{I_{0}\left(\sqrt{\kappa^{2}-\zeta^{2}-\varrho^{2}-2 \zeta \varrho+j 2 \kappa(\zeta+\varrho) \cos \alpha}\right)}{I_{0}(\kappa)},
\end{gathered}
$$

where $\zeta=2 \pi f_{D} \tau$, and $\varrho=2 \pi(a-b) \Delta$. We assume the same Rician factor $K$ and unit channel power $E\left[\left|h_{l}(t)\right|^{2}\right]=1$ at each element. Noise components are independent at different elements, with the same power $\sigma_{v}^{2}$. It has been shown that the angle spread at the BS is generally 
small for macrocells in urban, suburban, and rural areas, most often less than $30^{\circ}$ [21], which corresponds to $\kappa \geq 14.6$. For such scenarios, the spatial channel cross-correlation function $r_{h}((b-a) \Delta, 0)=E\left[h_{a}(t) h_{b}^{*}(t)\right], 1 \leq a<b \leq L$, can be accurately approximated by [21]

$$
r_{h}((b-a) \Delta, 0) \approx\left\{\frac{e^{-\frac{\varrho^{2} \sin ^{2} \alpha}{2 \kappa}}}{K+1}+\frac{K}{K+1}\right\} e^{j \varrho \cos \alpha} .
$$

Note that in (32), $\alpha_{0}=\alpha$ is assumed, due to the small angle spread at the BS.

With $\tau=0$ and $a \neq b$ in (30), the time-varying spatial cross-correlation function in (30) can be written as

$$
r_{z}((b-a) \Delta,(t ; 0))=r_{h}((b-a) \Delta, 0) r_{s}(t ; 0)
$$

where $r_{h}((b-a) \Delta, 0)$ for macrocells is given in (32) and $r_{s}(t ; 0)$ can be obtained from (5). Since noise components of different elements are independent, the effect of noise has not shown up in (33) as $a \neq b$. As we will see later in (34), $\cos \alpha$ can be estimated by looking at the phase of $r_{z}((b-a) \Delta,(t ; 0))$ in (33), due to the special form of $r_{h}((b-a) \Delta, 0)$ in (32) and also because $r_{s}(t ; 0)$ in (33) is real and positive.

When the BS experiences such heavy nonisotropic scattering $\kappa \geq 14.6$, it is straightforward to verify that there is a strong peak in the power spectrum of each branch $\Omega_{h_{l}}(f)$, at $f_{D} \cos \alpha$ [16]. On the other hand, based on (15), it can be shown that $\Omega_{s}(1 ; f)$, for a zero-mean i.i.d. sequence $\{w(m)\}$, is a uni-modal spectrum centered at $f=1 /(2 T)$, with a bandwidth of $\beta / T$. In addition, for the training sequence $w(m)=(-1)^{m} \sigma_{w}$, one can see from (24) that $\Omega_{h_{l}}(f)$ is an impulse at $f=1 /(2 T)$ as well. Therefore, according to (27) and due to the impulsive shape of $\Omega_{h_{l}}(f)$ when $\kappa$ is large, we conclude that the peak of $\Omega_{z_{l}}(1 ; f)$ for each branch happens at $f=f_{D} \cos \alpha+1 /(2 T)$. Now relying upon both cyclic spectrum and the spatial information provided by multiple antennas, we propose the following space-time estimator.

Based on (33), we can estimate $\cos \alpha$ via

$$
\widehat{\cos \alpha} \approx \measuredangle \hat{R}_{z}(\Delta,(0 ; 0)) /(-2 \pi \Delta)
$$

where $\measuredangle$ denotes the phase of a complex number and $\hat{R}_{z}(\Delta,(0 ; 0))$ is the estimate of $R_{z}(\Delta,(k ; 0))=$ $T^{-1} \int_{0}^{T} r_{z}(\Delta,(t ; 0)) \exp \{-j 2 \pi k t / T\} d t$ with $k=0$ and $r_{z}(\Delta,(t ; 0))=E\left[z_{a}(t) z_{a+1}^{*}(t)\right], \forall a \in$ $[1, L-1]$. We also have $\hat{R}_{z}(\Delta,(0 ; 0))=(L-1)^{-1} \sum_{l=1}^{L-1} \hat{R}_{z}^{l}(\Delta,(0 ; 0))$ where $\hat{R}_{z}^{l}(\Delta,(0 ; 0))=$ $N^{-1 / 2} \sum_{n=0}^{N-1} z_{l}(n) z_{l+1}^{*}(n), l \in[1, L-1]$, is the $l$-th adjacent-antenna-pair estimate of $R_{z}(\Delta,(0 ; 0))$. It is worth emphasizing that if noise components $v_{l}(t), l=1,2, \ldots, L$ are spatially correlated, 
we need replace $\hat{R}_{z}(\Delta,(0 ; 0))$ in (34) with $\hat{R}_{z}(\Delta,(1 ; 0))$ in order to have a noise-free estimate of $\widehat{\cos \alpha}$. Finally, $f_{D}$ can be estimated via

$$
\hat{f}_{D}=\frac{P}{\widehat{\cos \alpha} L T} \sum_{l=1}^{L}\left|-\frac{1}{2 T}+\arg \max _{f}\right| \hat{\Omega}_{z_{l}}(1 ; f)||,
$$

where $\hat{\Omega}_{z_{l}}(1 ; f)$ can be obtained via (28). Note that the cyclic-spectrum-based estimator in (35) can be applied either blindly or with the aid of the training sequence $w(m)=(-1)^{m} \sigma_{w}$.

\section{Simulation Results}

In this section, we first evaluate the performance of the proposed single antenna estimators using Monte Carlo simulation and next investigate the effect of noise, nonisotropic scattering, and LOS. Then we present the performance of our space-time estimator. The fading channel is generated using the spectral method [22]. The bandlimited Gaussian noise $v(t)$, with the autocorrelation $\sigma_{v}^{2} g(\tau)$, is simulated via the same method. It is worth noting that our algorithm does not put any constraint on the distribution of both the fading process and the noise, although only for simulation purposes we generate them as complex Gaussian processes. We define signalto-noise ratio $\mathrm{SNR}=\sigma_{w}^{2} / \sigma_{v}^{2}$. The performance of the estimator is measured by using the root mean squared error (RMSE) criterion $\left\{E\left[\left(\hat{f}_{D}-f_{D}\right)^{2}\right]\right\}^{1 / 2}$.

In all the simulations we have, the roll-off factor $\beta=0.5$, oversampling rate $P=8$, and the symbol duration $T=0.001$ second. Each data-aided estimation uses $M=256$ symbols and 200 Monte Carlo simulations, whereas blind algorithms use $M=512$ 4-QAM i.i.d. symbols 400 Monte Carlo simulations. The abbreviations DA, NDA, CC, and CS in the figures refer to data aided, non-data aided (blind), cyclic correlation, and cyclic spectrum, respectively. For example, DA-CC in a legend box represents a data-aided cyclic correlation based speed estimator.

We first investigate the performance of single antenna estimators, illustrated from Fig. 1 Fig. 6. Fig. 1 shows the performance of three estimators DA-CC, NDA-CC, and DA-CS when the channel is isotropic, $\kappa=0$, Rayleigh fading with $\mathrm{SNR}=10 \mathrm{~dB}$. Obviously, the DA-CS is the best, and DA-CC and NDA-CC have comparable estimation errors at small Dopplers, while DA-CC performs better than NDA-CC at large Dopplers. The robustness of these estimators against noise is shown in Fig. 2 in isotropic Rayleigh fading, for a fixed $f_{D} T=0.1$. Again, the DA-CS exhibits the best performance. Fig. 3 and Fig. 4 demonstrate the effect of nonisotropic scattering parameters $\kappa$ and $\alpha$. As one can see, the CS-based method is less sensitive to $\kappa$ than 


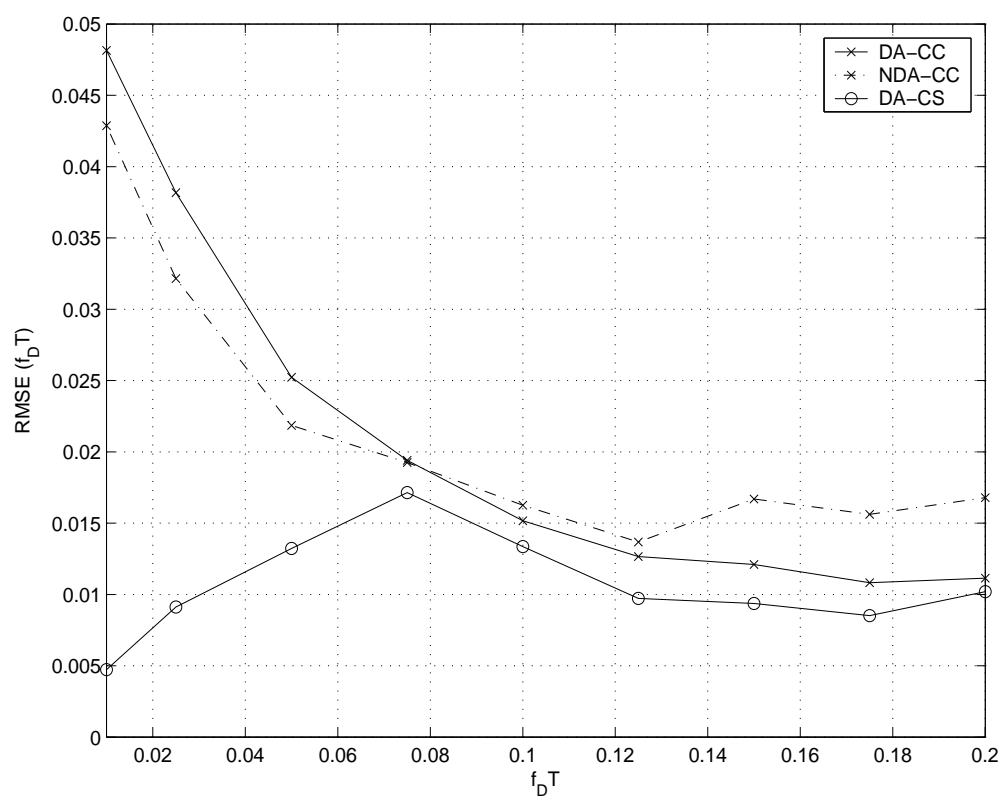

Fig. 1. RMSE versus $f_{D} T$ in isotropic Rayleigh fading, SNR $=10 \mathrm{~dB}$.

the CC-based techniques but more sensitive to $\alpha$. This is because for highly directive scenarios with $\alpha \rightarrow 90^{\circ}$, the strongest peak of $\Omega_{z}(1 ; f)$ moves away from $1 /(2 T) \pm f_{D}$. Based on Fig. 5 and Fig. 6, we have similar observation regarding the effect of the LOS parameters $K$ and $\alpha_{0}$. Note that for all the curves in Fig. 3 - Fig. 6, we have $f_{D} T=0.1$ and $\mathrm{SNR}=10 \mathrm{~dB}$.

Now we evaluate the performance of the space-time CS-based estimator in (35). In the simulation, $L=4$ space-time correlated complex Gaussian processes for the macrocell scattering scenario of $\kappa=100$ and $\alpha=60^{\circ}$ are generated, with $\Delta=1 / 2$. For each branch, the noise power is the same as the single antenna case, with $\mathrm{SNR}=10 \mathrm{~dB}$ per branch. Fig. 7 illustrates the estimation error, for both DA and NDA approaches. As we see, the DA-CS method provides excellent performance over a wide range of Dopplers. The curve for the DA-CS method for $L=1$ is also shown in Fig. 7, which clearly shows the advantage of using more than one antenna.

\section{CONCLUSION}

In this paper we have proposed methods for estimating the mobile speed using a linearly modulated waveform transmitted in a fading channel. The new methods exploit the cyclostationarity of the received signal. Two classes of algorithms based on the second order cyclic statistics, 


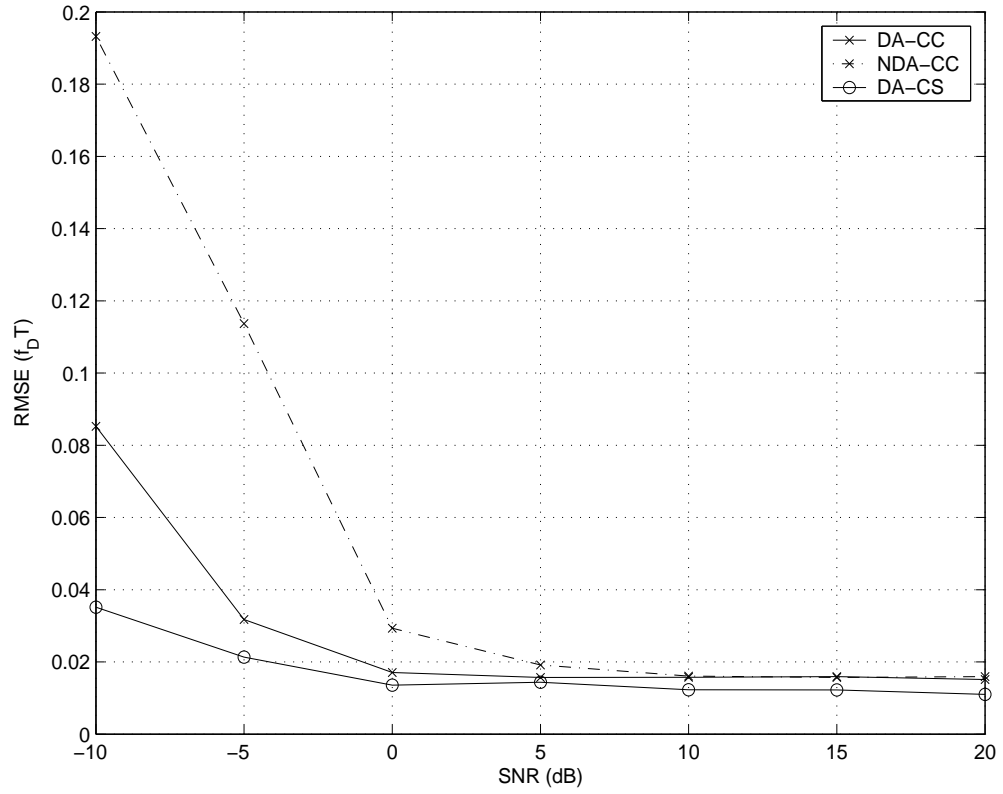

Fig. 2. RMSE versus SNR in isotropic Rayleigh fading, $f_{D} T=0.1$.

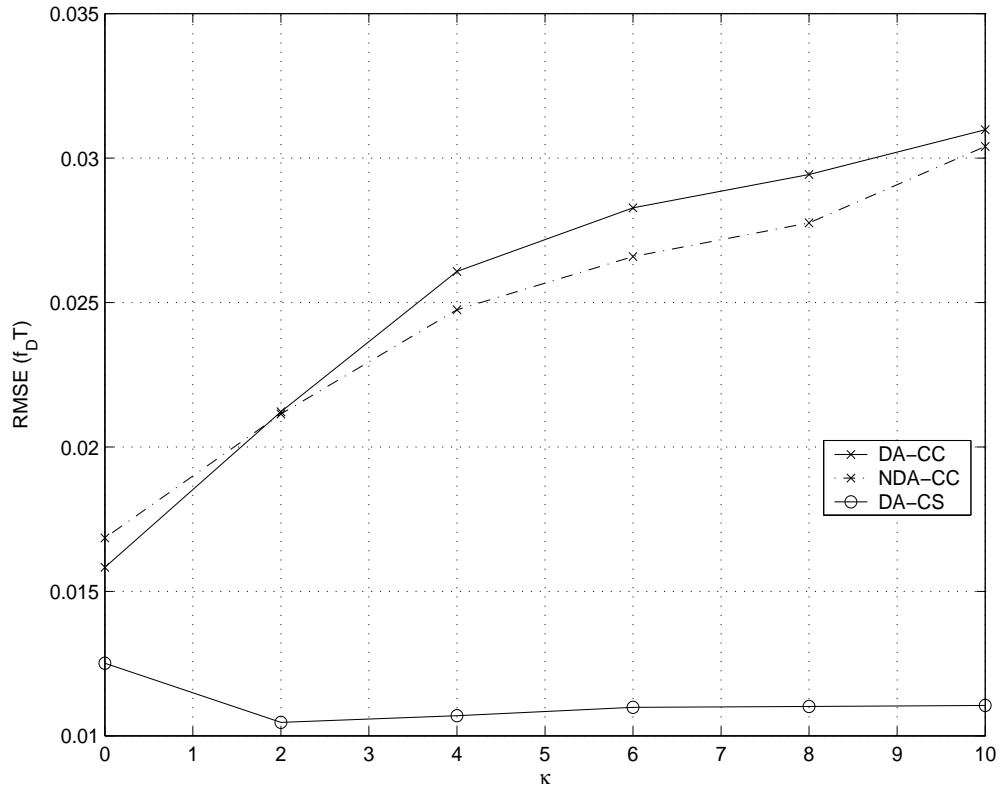

Fig. 3. RMSE versus the nonisotropic scattering parameter $\kappa$ in Rayleigh fading, $\mathrm{SNR}=10 \mathrm{~dB}, \alpha=30^{\circ}$, and $f_{D} T=0.1$. 


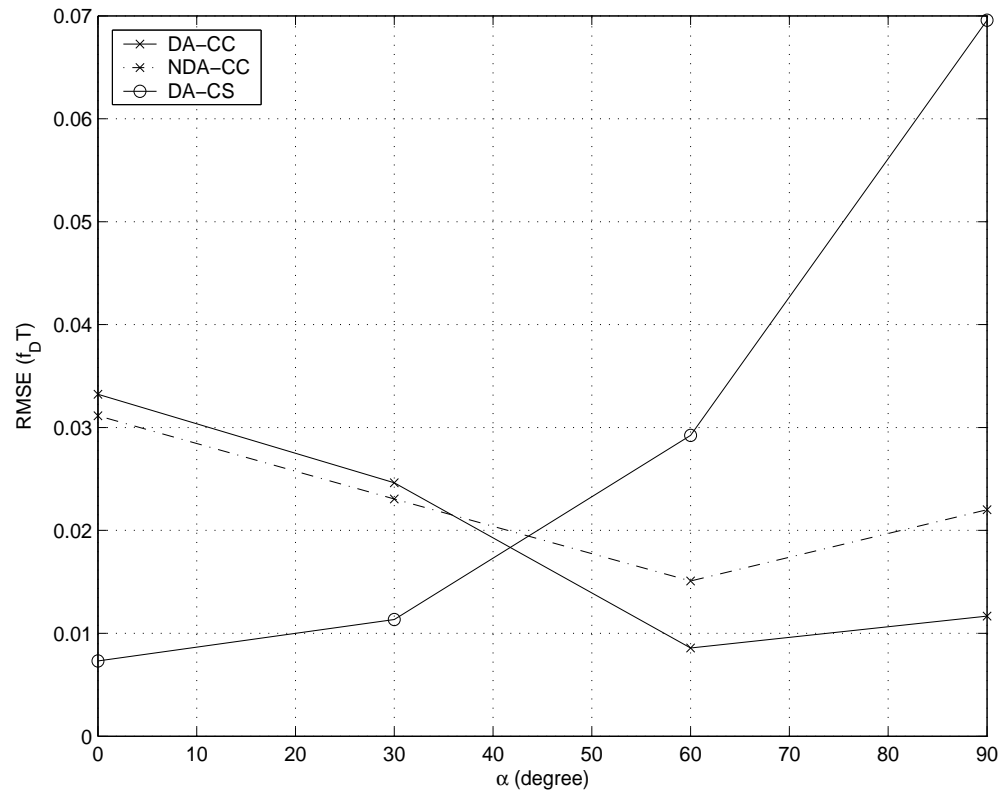

Fig. 4. RMSE versus the mean AOA $\alpha$ in Rayleigh fading, $\mathrm{SNR}=10 \mathrm{~dB}, \kappa=3.3$, and $f_{D} T=0.1$.

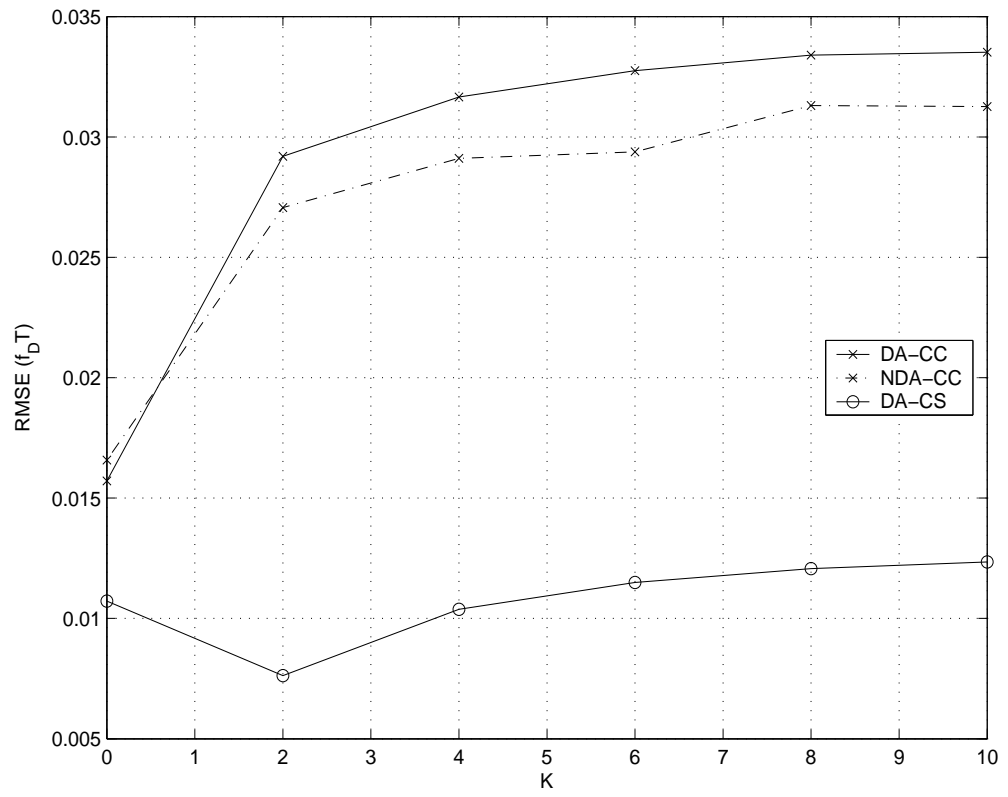

Fig. 5. RMSE versus the Rician factor $K$ in Rician isotropic fading, $\mathrm{SNR}=10 \mathrm{~dB}, \alpha_{0}=30^{\circ}$, and $f_{D} T=0.1$. 


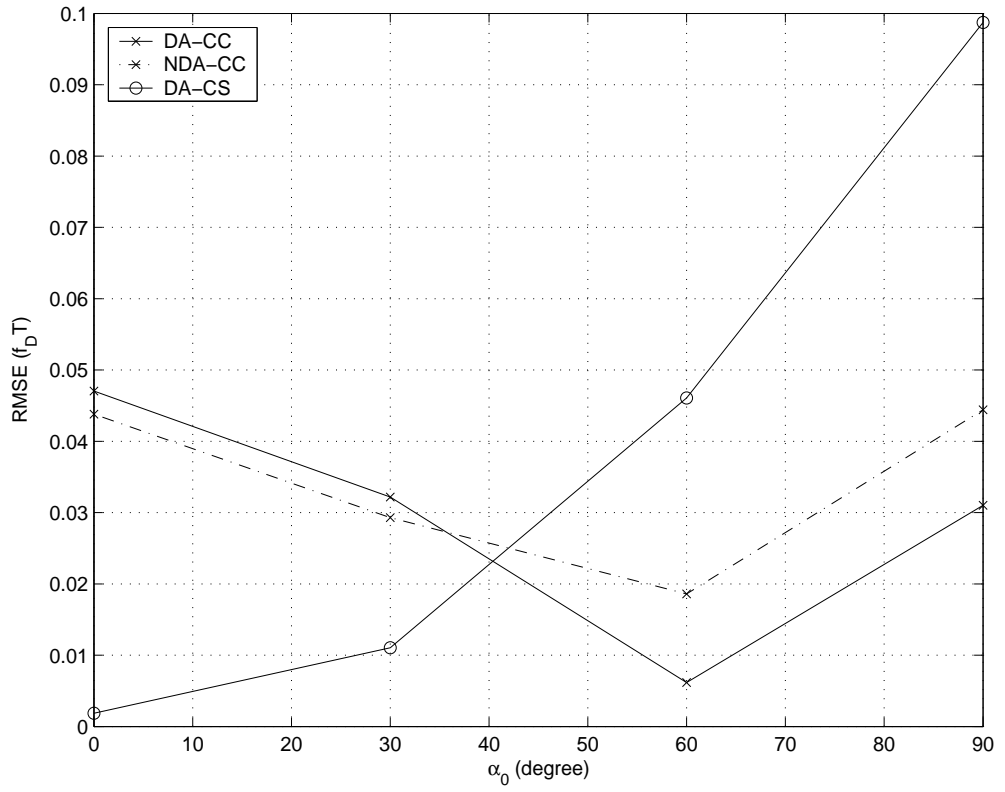

Fig. 6. RMSE versus the LOS AOA $\alpha_{0}$ in Rician isotropic fading, $\mathrm{SNR}=10 \mathrm{~dB}, K=5$, and $f_{D} T=0.1$.

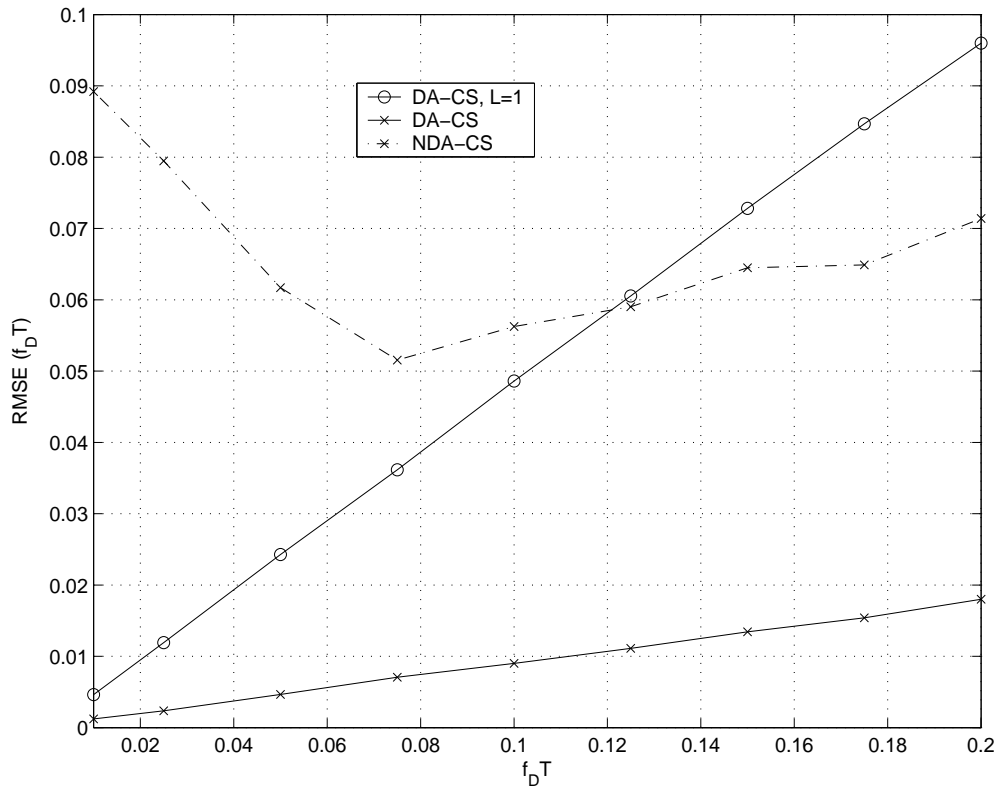

Fig. 7. RMSE versus $f_{D} T$ in Rayleigh fading, $\mathrm{SNR}=10 \mathrm{~dB}, \kappa=100, \alpha=60^{\circ}, L=4$, and $\Delta=1 / 2$. 
i.e., the cyclic correlation and cyclic spectrum, are developed. Comparing with the conventional pilot-tone-based speed estimation techniques, the resulting estimators provide several benefits, including robustness to stationary noise and interference, as well as cyclostationary signals with different cyclic frequencies. These algorithms can also be implemented blindly, which can increase the data throughput.

\section{APPENDIX I}

DERIVATION OF (24) AND (25)

For the deterministic training sequence $w(m)=(-1)^{m} \sigma_{w}, \tilde{r}_{w}(n-m)=E\left\{w(m) w^{*}(n)\right\}$ in (5) should be replaced by $w(m) w^{*}(n)$, which yields

$$
\begin{aligned}
r_{s}(t ; \tau) & =\sum_{m=-\infty}^{\infty} \sum_{n=-\infty}^{\infty} w(m) w^{*}(n) g(t-m T) g^{*}(t+\tau-n T) \\
& =\sigma_{w}^{2} \sum_{m=-\infty}^{\infty} \sum_{n=-\infty}^{\infty}(-1)^{(m+n)} g(t-m T) g^{*}(t+\tau-n T) \\
& =\sigma_{w}^{2} \sum_{l=-\infty}^{\infty}(-1)^{l} \sum_{m=-\infty}^{\infty} g(t-m T) g^{*}(t+\tau-m T-l T) \\
& =\sum_{l=-\infty}^{\infty} \tilde{r}_{T S}(l) \sum_{m-\infty}^{\infty} g(t-m T) g^{*}(t+\tau-m T-l T) .
\end{aligned}
$$

In the derivation we set $n=m+l$ and $\tilde{r}_{T S}(l)=w(m) w^{*}(n)=(-1)^{l} \sigma_{w}^{2}$, where TS stands for training sequence. By comparing (36) with (5) and then using (7), when such training sequence is transmitted, we obtain the cyclic spectrum of $s(t)$ as

$$
\Omega_{s}(k ; f)=\frac{1}{T} G(f) G^{*}\left(f-\frac{k}{T}\right) \tilde{\Omega}_{T S}(f),
$$

where

$$
\begin{aligned}
\tilde{\Omega}_{T S}(f) & =\sum_{l=-\infty}^{\infty} \tilde{r}_{s}(l) e^{-j 2 \pi l T f}=\sigma_{w}^{2} \sum_{l=-\infty}^{\infty}(-l)^{l} e^{-j 2 \pi l T f} \\
& =\sigma_{w}^{2} \sum_{l=-\infty}^{\infty} e^{j l \pi} e^{-j 2 \pi l T f} \\
& =\frac{\sigma_{w}^{2}}{T} \sum_{m=-\infty}^{\infty} \delta\left(f-\frac{m}{2 T}\right), m \text { odd },
\end{aligned}
$$

where Fourier transform of the complex exponential sequence $e^{j l \pi}[23$, p. 54] is used in the last step of derivation. Note that for the raised cosine pulse $g(t)$, we already know that $\Omega_{G}(k ; f) \triangleq$ 
$G(f) G^{*}\left(f-\frac{k}{T}\right) \equiv 0, \forall|k| \geq 2$ and $\Omega_{G}(1 ; f)$ is nonzero over $[(1-\beta) /(2 T),(1+\beta) /(2 T)]$. Furthermore, $\Omega_{G}(1 ;(1-\beta) /(2 T))=\Omega_{G}(1 ;(1+\beta) /(2 T))=0$. Therefore, (37) simplifies to

$$
\begin{aligned}
\Omega_{s}(1 ; f) & =\frac{\sigma_{w}^{2}}{T^{2}} \Omega_{G}(1 ; f) \delta\left(f-\frac{1}{2 T}\right) \\
& =\frac{\sigma_{w}^{2}}{4} \delta\left(f-\frac{1}{2 T}\right) .
\end{aligned}
$$

The Fourier transform of (39) w.r.t. $f$ leads us to (24).

Following the same procedure, it is easy to show that the cyclic spectrum of $\dot{s}(t)$ for $k=1$ is

$$
\begin{aligned}
\Omega_{\dot{s}}(k ; f) & =\frac{1}{T} \breve{G}(f) \breve{G}^{*}\left(f-\frac{k}{T}\right) \tilde{\Omega}_{T S}(f) \\
& =-\frac{4 \pi^{2} f^{2}}{T^{2}} G(f) G^{*}\left(f-\frac{k}{T}\right) \tilde{\Omega}_{T S}(f) \\
& =-\frac{\pi^{2} \sigma_{w}^{2}}{4 T^{2}} \delta\left(f-\frac{1}{2 T}\right)
\end{aligned}
$$

where $\breve{G}(f)$ is given in (19). Obviously, we obtain (25) after taking the Fourier transform of (40).

\section{REFERENCES}

[1] M. J. Chu and W. E. Stark, "Effect of mobile velocity on communications in fading channels," IEEE Trans. Veh. Technol., vol. 49, pp. 202-210, 2000.

[2] G. L. Stüber, Principles of Mobile Communication, 2nd ed. Boston, MA: Kluwer, 2001.

[3] A. Abdi, H. Zhang, and C. Tepedelenlioglu, "A unified approach to the performance analysis of speed estimation techniques in mobile communication,” IEEE Trans. Commun., vol. 56, pp. 126-135, 2008.

[4] J. Holtzman and A. Sampath, "Adaptive averaging methodology for handoffs in cellular systems," IEEE Trans. Veh. Technol., vol. 44, pp. 59-66, 1995.

[5] K. Anim-Appiah, "On generalized covariance-based velocity estimation,” IEEE Trans. Veh. Technol., vol. 48, pp. 1546$1557,1999$.

[6] C. Tepedelenlioglu and G. Giannakis, "On velocity estimation and correlation properties of narrow-band mobile communication channels," IEEE Trans. Veh. Technol., vol. 50, pp. 1039-1052, 2001.

[7] J. Lin and J. G. Proakis, "A parametric method for Doppler spectrum estimation in mobile radio channels," in Proc. Conf. Inf. Sciences and Systems, Baltimore, MD, 1993, pp. 875-880.

[8] K. E. Baddour and N. C. Beaulieu, "Robust Doppler spread estimation in nonisotropic fading channels," IEEE Trans. Wireless Commun., vol. 4, pp. 2677-2682, 2005.

[9] L. Krasny, H. Arslan, D. Koilpillai, and S. Chennakeshu, "Doppler spread estimation in mobile radio systems," IEEE Commun. Lett., vol. 5, pp. 197-199, 2001.

[10] A. Dogandzic and B. Zhang, "Estimating Jakes' Doppler power spectrum parameters using the Whittle approximation," IEEE Trans. Signal Processing, vol. 53, pp. 987-1005, 2005. 
[11] K. Baddour and N. Beaulieu, "Nonparametric Doppler spread estimation for flat fading channels," in IEEE Wireless Commun. and Networking Conf., New Orleans, LA, 2003, pp. 953-958.

[12] S. Mohanty, "VEPSD: A novel velocity estimation algorithm for next-generation wireless systems," IEEE Trans. Wireless Commun., vol. 4, pp. 2655-2660, 2005.

[13] H. Zhang and A. Abdi, "A robust mobile speed estimator in fading channels: Performance analysis and experimental results," in Proc. IEEE Global Telecommun. Conf., St. Louis, MO, 2005, pp. 2569-2573.

[14] M. Ghogho, A. Swami, and T. Durrani, "Blind synchronization and Doppler spread estimation for MSK signals in time-selective fading channels," in Proc. IEEE Int. Conf. Acoust., Speech, Signal Processing, Istanbul, Turkey, 2000, pp. 2665-2668.

[15] J. G. Proakis, Digital Communications, 4th ed. New York: McGraw-Hill, 2001.

[16] A. Abdi, J. A. Barger, and M. Kaveh, "A parametric model for the distribution of the angle of arrival and the associated correlation function and power spectrum at the mobile station," IEEE Trans. Veh. Technol., vol. 51, pp. 425-434, 2002.

[17] W. A. Gardner, Introduction to Random Processes: With Applications to Signals and Systems, 2nd ed. New York: McGraw-Hill, 1990.

[18] F. D. Neeser and J. L. Massey, "Proper complex random processes with applications to information theory," IEEE Trans. Inform. Theory, vol. 39, pp. 1293-1302, 1993.

[19] A. V. Dandawate and G. B. Giannakis, "Nonparametric polyspectral estimators for kth-order (almost) cyclostationary processes," IEEE Trans. Inform. Theory, vol. 40, pp. 67-84, 1994.

[20] J. Choi, "Mimo equalization for space-time coded signals using cyclostationarity," in Proc. IEEE Veh. Technol. Conf., Orlando, FL, 2003, pp. 272-276.

[21] A. Abdi and M. Kaveh, "Parametric modeling and estimation of the spatial characteristics of a source with local scattering," in Proc. IEEE Int. Conf. Acoust., Speech, Signal Processing, Orlando, FL, 2002, pp. 2821-2824.

[22] K. Acolatse and A. Abdi, "Efficient simulation of space-time correlated MIMO mobile fading channels," in Proc. IEEE Veh. Technol. Conf., Orlando, FL, 2003, pp. 652-656.

[23] A. V. Oppenheim, R. W. Schafer, and J. R. Buck, Discrete-Time Signal Processing, 2nd ed. Upper Saddle River, NJ: Prentice Hall, 1998. 